\title{
Eventos adversos em unidades de terapia intensiva adulto: uma revisão integrativa
}

\author{
Adverse events in adult intensive care units: an integrative review \\ Eventos adversos en unidades de cuidados intensivos para adultos: una revisión integradora
}

Recebido: 21/07/2021 | Revisado: 26/07/2021 | Aceito: 28/07/2021 | Publicado: 04/08/2021

\author{
Marcela Claudia de Paula Oliveira \\ ORCID: https://orcid.org/0000-0002-7762-0684 \\ Universidade de Pernambuco, Brasil \\ E-mail: marcela-claudia2011@hotmail.com \\ Jeniffer Bezerra Guimarães Barreto \\ ORCID: https://orcid.org/0000-0002-3250-4575 \\ Universidade Federal de Pernambuco, Brasil \\ E-mail: jeniffbar@hotmail.com \\ Virgínia Menezes Coutinho \\ ORCID: https://orcid.org/0000-0001-9063-758X \\ Universidade Federal de Pernambuco, Brasil \\ E-mail: vi.menezes@hotmail.com \\ Patrícia da Costa Araújo Magalhães \\ ORCID: https://orcid.org/0000-0003-1058-6503 \\ Universidade Federal de Pernambuco, Brasil \\ E-mail: patricia_costa145@hotmail.com
}

\begin{abstract}
Resumo
Os cuidados em uma Unidade de terapia intensiva (UTI) são destinados aos pacientes críticos, que pela sua complexidade são mais expostos à ocorrência de eventos adversos (EA), geralmente decorrentes da assistência à saúde como as flebites, erros de medicação e a Pneumonia associada a ventilação mecânica. Estes EA podem causar danos temporários ou permanentes e a sua ocorrência está atrelada a múltiplos fatores relativos ao paciente e processos de trabalho. Objetivou Identificar em publicações cientifica nacionais e internacionais evidências a respeito dos eventos adversos mais comuns em Unidades de Terapia Intensiva adulto. Revisão integrativa realizada nas bases de dados Mediline, Scopus,Scielo e Lilacs entre abril e maio de 2021 com recorte temporal de 2016 a 2021. Foram incluídos 10 artigos publicados no idioma inglês e português. Os resultados apontaram que os Eventos adversos mais frequentes estão atrelados aos cuidados de saúde, destacando os processos/procedimentos clínicos e que há fatores que influenciam na ocorrência dos eventos como: a idade, comorbidades, gravidade, maior carga de trabalho da enfermagem, desproporcional dimensionamento de pessoal. Embora os eventos adversos sejam considerados um problema de saúde pública bem presente nas unidades de terapia intensiva, ainda existem poucos estudos sobre a temática.
\end{abstract}

Palavras-chave: Eventos adversos; Unidade de terapia intensiva.

\begin{abstract}
Care in an Intensive Care Unit (ICU) is intended for critically ill patients, who, due to their complexity, are more exposed to the occurrence of adverse events (AE), usually resulting from health care such as phlebitis, medication errors and Pneumonia associated with mechanical ventilation. These AEs can cause temporary or permanent damage and their occurrence is linked to multiple factors related to the patient and work processes. It aimed to identify scientific evidence on the most frequent adverse events in ICUs. Integrative review performed in Mediline, Scopus, Scielo and Lilacs databases between April and May 2021 with a time frame from 2016 to 2021. 10 articles published in English and Portuguese were included. The results showed that the most frequent adverse events are linked to health care, highlighting the clinical processes/procedures and that there are factors that influence the occurrence of events such as: age, comorbidities, severity, greater nursing workload, disproportionate staffing. Although adverse events are considered a public health problem that is very present in intensive care units, there are still few studies on the subject.
\end{abstract}

Keywords: Adverse events; Intensive care unit.

\section{Resumen}

La atención en una unidad de cuidados intensivos (UCI) está destinada a pacientes críticamente enfermos, que por su complejidad están más expuestos a la ocurrencia de eventos adversos (EA), generalmente derivados de cuidados de salud como flebitis, errores de medicación y neumonía asociada. Ventilacion mecanica. Estos EA pueden provocar daños temporales o permanentes y su aparición está vinculada a múltiples factores relacionados con el paciente y los procesos laborales. Su objetivo fue identificar evidencia en publicaciones científicas nacionales e internacionales 
sobre los eventos adversos más comunes en Unidades de Cuidados Intensivos de adultos. Revisión integrativa realizada en las bases de datos Mediline, Scopus, Scielo y Lilacs entre abril y mayo de 2021 con un marco temporal de 2016 a 2021. Se incluyeron 10 artículos publicados en inglés y portugués. Los resultados mostraron que los eventos adversos más frecuentes están vinculados al cuidado de la salud, destacando los procesos / procedimientos clínicos y que existen factores que influyen en la ocurrencia de eventos como: edad, comorbilidades, severidad, mayor carga de trabajo de enfermería, dimensionamiento desproporcionado del personal. Si bien los eventos adversos se consideran un problema de salud pública muy presente en las unidades de cuidados intensivos, aún existen pocos estudios sobre el tema.

Palabras clave: Eventos adversos; Unidad de cuidados intensivos.

\section{Introdução}

Os cuidados em uma Unidade de Terapia Intensiva (UTI) são destinados aos pacientes críticos, que carecem de cuidados contínuos, pela criticidade e instabilidade hemodinâmica que apresentam (ROQUE et al. 2016). Diante da complexidade e da grande demanda de procedimentos e dispositivos invasivos, estes pacientes estão mais expostos à ocorrência de eventos adversos decorrentes de erros e falhas assistenciais (Merino et al. 2012; Pagnamenta et al. 2012; Roque et al. 2016;).

Os Eventos Adversos (EA), de acordo com a Organização Mundial da Saúde (OMS), são incidentes evitáveis que atingem ao paciente, podendo causar danos temporários ou permanentes, leves a graves e são geralmente decorrentes da assistência à saúde. Estes danos produzem prejuízos psicológicos e até mesmo o óbito (Brasil, 2015, 2013; Lima \& Barbosa, 2015; Roque et al. 2016).

A OMS verificou que ocorrência de incidentes relacionados à assistência à saúde, e em particular de EA, afeta de $4,0 \%$ a $16 \%$ de pacientes hospitalizados nos países desenvolvidos (WHO, 2008). Em um estudo realizado de 2007 a 2011 com dados epidemiológicos da OMS, verificou-se que um total de 421 milhões de hospitalizações ocorre no mundo anualmente e dessas, 42,7 milhões de eventos adversos relacionados ao cuidado em saúde (Jha et al. 2013; Françolin et al., 2015).

No Brasil foram notificados e confirmados pelo Notivisa e FormSUS no período de 2014 a 2016, 63.933 eventos adversos relacionados com a assistência à saúde, em que $417(0,6 \%)$ tiveram como consequência o óbito. Os estados que registraram óbito foram Alagoas, Paraíba, Pernambuco, Rondônia, Roraima, Bahia e Mato Grosso. E com relação à ocorrência desses eventos na Unidade de Terapia Intensiva, foram notificados 141 casos (Maia et al. 2018).

Estes eventos de acordo com a OMS podem ser classificados conforme a origem ou tipo do evento ocorrido em processo/procedimento clínico como as lesões por pressão, complicação na intubação orotraqueal; quedas; infecções relacionadas à assistência à saúde, como as infecções da corrente sanguínea, infecção do trato respiratório e urinário; os referentes à medicação, dispositivos médicos/equipamento médico (falha/avaria de equipamento) e infraestrutura (Brasil, 2017; Roque et al. 2016).

Existem alguns fatores de risco atrelados ao paciente que propiciam o acontecimento dos EA, dentre eles se destacam: a idade avançada, a alteração no nível de consciência, que pode ser avaliado pela escala de Glasgow e a presença de comorbidades, como um importante fator preditor de complicações e gravidade que pode ser avaliado pelo score APACHE II (Acute Physiology and Chronic Health Evalution) (Knaus et al. 1981).

Nazareno et al.(2010) retrata que há outros fatores que também merecem destaque são o uso de múltiplos fármacos, o longo tempo de permanência nas instituições de saúde, a realização de muitos procedimentos invasivos, prolongando tempo de internação hospitalar e surgimento de novas infecções. Contribuem também para a ocorrência dos EA aspectos relacionados ao profissional de saúde tais como as falhas humanas associadas a sobrecarga de trabalho, baixa remuneração e alto nível de estresse (Souza et al. 2012; Novarreti et al. 2014;).

No Brasil entrou em vigor em 10 de abril de 2013 a Portaria 529, que versa sobre a Política Nacional de Segurança do Paciente (PNSP) e a RDC 36 que declara ações para segurança do paciente nos serviços de saúde, para promover práticas 
assistenciais seguras através da lavagem das mãos, identificação do paciente, comunicação efetiva, assim como prevenção de quedas, lesão por pressão e segurança nas medicações (Brasil, 2015, 2013). Nesse contexto, as instituições de saúde implantaram os Núcleos de Segurança do Paciente (NSP) que analisa os EA relacionados à assistência à saúde, notifica no Sistema Nacional de Vigilância Sanitária (SNVS) cujo objetivo é criar e executar planos de ações direcionados ao evento e aos profissionais como forma de prevenção destas ocorrências (Runciman, 2009; Françolin et al. 2015).

Tendo em vista esta problemática e sabendo que os eventos adversos (EA) são preveníveis, é percebida a relevância desta temática, uma vez que, quando os mesmos são identificados e notificados, são desenvolvidas estratégias de ações para a melhoria dos processos de trabalho. Frente ao exposto, o presente estudo teve como objetivo identificar as evidências científicas sobre os eventos adversos em UTIs adulto e responder ao questionamento: Quais são os eventos adversos mais frequentes nas Unidades de terapia intensiva adulto?

\section{Metodologia}

Trata-se de um estudo de revisão integrativa, de abordagem qualitativa, cujo método é direcionado por uma pergunta norteadora definida previamente, que reúne conhecimentos/resultados amplos sobre uma determinada problemática já discutida. Após a busca destes estudos, quando comparados, permite reunir as evidências científicas que corroboram para melhoria da prática assistência de saúde (Whittemore et al. 2005).

A construção da revisão integrativa foi constituída por 5 etapas: 1-identificação do tema e questão de pesquisa; 2busca na literatura com os critérios de inclusão e exclusão, 3- Avaliação dos estudos; 4- Análise e interpretação dos resultados; 5- apresentação da revisão/síntese do conhecimento (Crosseti, 2012).

A pergunta norteadora foi elaborada pela técnica PICO que corresponde a: Problema: Eventos adversos em UTIs; C: (Intervenção padrão) Medidas de prevenção dos Eventos adversos. Os ítens O: (Resultados esperados) e I: (intervenção de interesse) não foram objetivos do presente estudo. Dessa maneira a pergunta norteadora foi: Quais os eventos adversos mais comuns em Unidades de Terapia Intensiva adulto?

Os critérios de inclusão foram: estudos completos e originais completos na íntegra que retratassem a temática, nos idiomas: português e inglês, dos últimos 5 anos (janeiro do ano de 2016 a maio de 2021). Foram excluídas teses, dissertações, editoriais capítulos de livros, revisões integrativas e narrativas, relatos de experiência.

O quantitativo de publicações encontradas foi possível pelo cruzamento dos descritores (Quadro 1). A busca foi realizada nos meses de abril e maio de 2021 nas seguintes bases de dados: Medical literature Analysis and Retrieval System Online (MEDILINE) via PUBMED, SCOPUS e Literatura Latino Americana e do Caribe em ciências da saúde (LILACS) e Scientific Electronic Library Online (Scielo). Os descritores utilizados foram buscados no Descritores em ciências da saúde (DECS) e no Medicall Subject Headings section (MESH) em associação com operador booleano AND. Os descritores utilizados foram: eventos adversos/adverse events; unidade de cuidados intensivos/unit care intensive.

Quadro 1. Publicações identificadas nas bases de dados no período de abril a maio de 2021.

\begin{tabular}{|l|c|c|c|c|c|}
\hline \multicolumn{1}{|c|}{$\begin{array}{c}\text { CRUZAMENTO DE } \\
\text { DESCRITORES }\end{array}$} & $\begin{array}{c}\text { MEDLINE/ } \\
\text { PUBMED }\end{array}$ & SCOPUS & Scielo & LILACS & Total \\
\hline $\begin{array}{l}\text { Eventos adversos/adverse events } \\
\text { AND Unidade de cuidados } \\
\text { intensivos/intensive care unit }\end{array}$ & 6.285 & 7.809 & 07 & 164 & 14.265 \\
\hline Total & 6.285 & 7.809 & 07 & 164 & 14.265 \\
\hline
\end{tabular}

Fonte: Autores, Recife (2021). 
Os cruzamentos dos descritores resultaram em 14.265 publicações. Todos os títulos dessas publicações foram lidos e então excluídos 12.839 estudos, por não estarem relacionados à temática abordada. Procedeu-se a leitura dos resumos dos 1.426 artigos restantes. Destes, 1.070 foram descartados, resultando em 356 publicações para leitura na íntegra. Diante de uma leitura rigorosa 343 estudos foram excluídos, por não responderem à pergunta norteadora, estarem duplicados ou não obedecerem aos critérios de inclusão estabelecidos. Assim foram incluídos na revisão integrativa 10 artigos científicos (Figura $1)$.

Figura 1. Fluxograma PRISMA dos estudos selecionados. Recife, 2021.

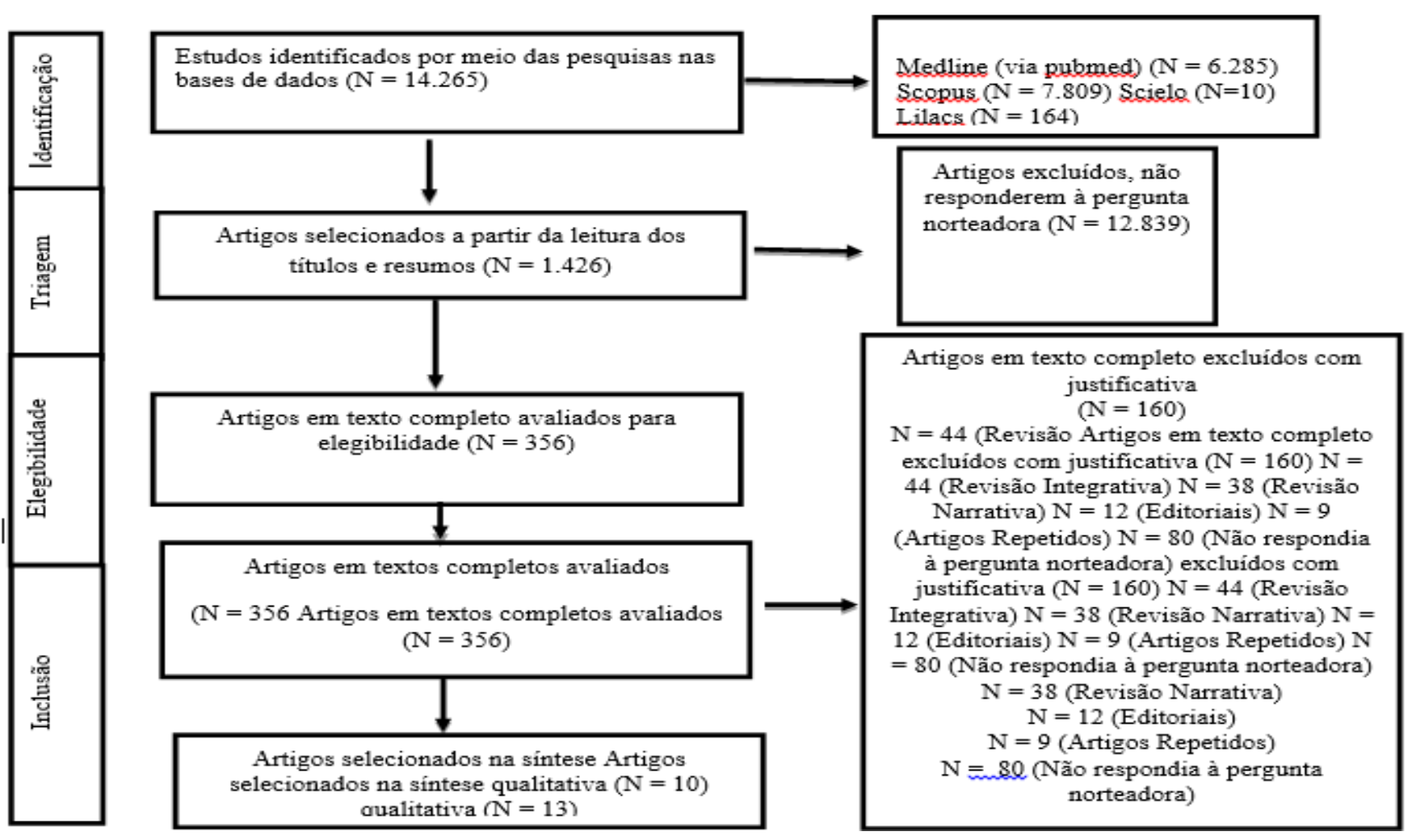

Fonte: Autores, Recife (2021).

A fim de se realizar uma análise metodológica rigorosa dos artigos, foi utilizado o instrumento adaptado do Critical Appraisal Skill Programme (CASP, 2013) que avalia dez itens contidos no artigo a ser estudado: objetivo, metodologia, discussão, seleção da amostra, dados que foram coletados, relação entre os pesquisadores, aspectos éticos preservados, análise de dados rigorosa e fundamentada, apresentação e discussão dos resultados, contribuições, limitações e indicações de novas questões de pesquisa.

Dessa maneira é dado um valor de zero ou um a cada ítem, sendo o máximo dez pontos. Mediante a pontuação atribuída o artigo se classifica em nível A: seis a dez pontos (boa qualidade metodológica e viés reduzido) e Nível B: no mínimo cinco pontos (qualidade metodológica satisfatória, porém com risco de viés aumentado) (CASP, 2013).

A fim de coletar e organizar os dados, foi utilizado um instrumento adaptado e validado pelas autoras que contemplam os dados: identificação da publicação, objetivo do estudo, método, nível de evidência, resultados (Pompeo, Rossi \& Galvão, 2009).

As pesquisas incluídas na revisão foram analisadas quanto ao nível de evidência científica segundo Oxford Centre for Evidence-based Medicine (2009), onde este classifica os desenho de estudo em 1A (Revisão sistemática de ensaios clínicos 
controlados randomizados), 1B (Ensaio clínico controlado randomizado com intervalo de confiança estreito), 1C (Resultados terapêuticos do tipo "tudo ou nada"), 2A (Revisão Sistemática de Estudos de Coorte), 2B (Estudo de Coorte), 2C (Observação de resultados terapêuticos/ Estudo Ecológico), 3A (Revisão Sistemática de Estudos Caso-Controle), 3B (Estudo Caso-Controle), 4 (Relatos de Caso) e 5 (Opinião de especialistas desprovida de avaliação crítica ou baseada em matérias básicas) (Oxford, 2009).

\section{Resultados e Discussão}

Foram analisados 10 artigos na íntegra no presente estudo, estes foram publicados nos idiomas inglês e português nos últimos 5 anos (Janeiro de 2016 a março de 2021). Em relação a bases de dados, quatro foram encontrados na Mediline/PUBMED, extraídos da SCIELO quatro, um da LILACS, um na SCOPUS. Conforme desenho de pesquisa dos estudos avaliados quatro estudos de coorte, um convergente assistencial; dois quantitativos; dois qualitativos, um quantiqualitativo. De acordo com rigor metodológico dos artigos selecionados foram avaliados pelo CASP, obtivemos artigos com nível A e B. O nível de evidência foi classificado conforme Oxford (2009). O nível dos estudos selecionados se classificou em $2 \mathrm{~B}$ e $2 \mathrm{C}$. 
Quadro 2. Distribuição das publicações na revisão integrativa de acordo com a autoria, ano de publicação, base de dados, estado, tipo de estudo, nível de evidência. Recife-PE, Brasil. 2021.

\begin{tabular}{|c|c|c|c|c|}
\hline AUTOR/ANO & $\begin{array}{l}\text { BASE DE } \\
\text { DADOS }\end{array}$ & LOCAL & TIPO DE ESTUDO & $\begin{array}{l}\text { NÍVEL DE } \\
\text { EVIDÊNCIA }\end{array}$ \\
\hline $\begin{array}{l}\text { Mansouri et al., } \\
2019 . \\
\end{array}$ & PUBMED & Irã. & $\begin{array}{c}\text { Analítico descritivo } \\
\text { (Qualitativo) }\end{array}$ & $2 \mathrm{C}$ \\
\hline $\begin{array}{l}\text { Teodoro RFB, Silva AS, } \\
\text { Carreiro MA et al.2020 }\end{array}$ & LILACS & $\begin{array}{l}\text { Niterói, Rio de Janeiro (RJ). } \\
\text { Brasil. }\end{array}$ & Quantitativo, transversal & $2 \mathrm{C}$ \\
\hline $\begin{array}{c}\text { Rodriguez et AL., } \\
2020 . \\
\text { PUBMED } \\
\end{array}$ & PUBMED & Valladolid, Espanha. & $\begin{array}{l}\text { Coorte prospectivo e } \\
\text { multicêntrico }\end{array}$ & $2 \mathrm{~B}$ \\
\hline SILVA et AL.,2018. & SCOPUS & Minas Gerais (MG). Brasil. & $\begin{array}{l}\text { Descritivo, } \\
\text { quantitativo, } \\
\text { prospectivo }\end{array}$ & $2 \mathrm{~B}$ \\
\hline Dodek et AL.,2019. & PUBMED & Vancouver, Canadá. & $\begin{array}{c}\text { Observacional } \\
\text { (Quanti-qualitativo) }\end{array}$ & $2 \mathrm{C}$ \\
\hline $\begin{array}{c}\text { Veiga VC, Postalli NF, } \\
\text { Alvarisa TK, Travassos PP, } \\
\text { Vale RT, Oliveira CZ, et al. } \\
2019\end{array}$ & SCIELO & $\begin{array}{c}\text { Bela vista, São Paulo (SP), } \\
\text { Brasil. }\end{array}$ & Coorte & $2 \mathrm{~B}$ \\
\hline $\begin{array}{c}\text { Maldonado, Ximena } \\
\text { Alejandra; Navarro } \\
\text { Nascimento, Eliane Regina } \\
\text { Pereira do; Lzarri, Daniele } \\
\text { Delacanal. 2020 }\end{array}$ & SCIELO & Magallanes, Chile. & $\begin{array}{c}\text { Pesquisa Convergente } \\
\text { Assistencial }\end{array}$ & $2 \mathrm{C}$ \\
\hline Roque KE et al. 2016 & PUBMED & $\begin{array}{l}\text { Cidade universitária, Rio de } \\
\text { Janeiro(RJ). Brasil. }\end{array}$ & Coorte concorrente & $2 \mathrm{~B}$ \\
\hline $\begin{array}{l}\text { Toffoletto MC, et AL. } \\
2016 \\
\end{array}$ & SCIELO & São Paulo(SP). Brasil. & Coorte retrospectivo & $2 \mathrm{~B}$ \\
\hline $\begin{array}{c}\text { Costa TD, Salvador PTCO, } \\
\text { Rodrigues CCFM, Alves } \\
\text { KYA, Tourinho FSV, } \\
\text { Santos VEP. } \\
2016\end{array}$ & SCIELO & $\begin{array}{l}\text { Rio Grande do Norte. Natal. } \\
\text { Brasil. }\end{array}$ & Qualitativo & $2 \mathrm{C}$ \\
\hline
\end{tabular}

Fonte: Autores 
Quadro 3. Distribuição das publicações na revisão integrativa de acordo com a autoria, objetivos e resultados. Recife-PE, Brasil. 2021.

\begin{tabular}{|c|c|c|}
\hline AUTORES/ ANO & OBJETIVO & RESULTADOS \\
\hline $\begin{array}{l}\text { Mansouri et al., } \\
2019 .\end{array}$ & $\begin{array}{l}\text { Avaliar a visão dos enfermeiros sobre as } \\
\text { principais barreiras para a notificação de erros e } \\
\text { eventos adversos em unidades de terapia } \\
\text { intensiva. }\end{array}$ & $\begin{array}{l}\text { Identificou três áreas principais que impediram o relato } \\
\text { de incidentes-medo das consequências, barreiras } \\
\text { processuais e barreiras de gerenciamento. }\end{array}$ \\
\hline $\begin{array}{l}\text { Teodoro RFB, Silva AS, } \\
\text { Carreiro MA et al., } \\
2020 .\end{array}$ & $\begin{array}{l}\text { Analisar os resultados da Pesquisa de Cultura } \\
\text { de Segurança do Paciente aos profissionais de } \\
\text { saúde sobre notificação de eventos adversos da } \\
\text { Unidade de terapia intensiva. }\end{array}$ & $\begin{array}{l}\text { Menos de } 45 \% \text { dos participantes da pesquisa sempre } \\
\text { notificam um erro, engano ou falha, que afete ou não o } \\
\text { paciente, } 59,0 \% \text { não fizeram nenhuma notificação nos } \\
\text { últimos } 12 \text { meses antecedentes à pesquisa. }\end{array}$ \\
\hline $\begin{array}{l}\text { Rodriguez et al., } \\
2020 .\end{array}$ & $\begin{array}{l}\text { Analisar se a utilização de escores de alerta } \\
\text { precoce pode auxiliar na tomada de decisão } \\
\text { determinando os pacientes que necessitam de } \\
\text { unidade de terapia intensiva }\end{array}$ & $\begin{array}{l}\text { Estudo incluiu um total de } 209 \text { casos. O total de } \\
\text { internações em unidades de terapia intensiva foi de } 50 \\
\text { casos }(23,9 \%) \text {. O uso de pontuações de alerta precoce } \\
\text { pode ajudar os serviços médicos de emergência como } \\
\text { ferramentas para a detecção precoce de eventos } \\
\text { adversos graves e, portanto, atuam como preditores do } \\
\text { risco de deterioração }\end{array}$ \\
\hline $\begin{array}{l}\text { SILVA et AL., } \\
2018 .\end{array}$ & $\begin{array}{l}\text { Identificar os incidentes e eventos adversos no } \\
\text { transporte intra-hospitalar em terapia intensiva }\end{array}$ & $\begin{array}{l}\text { A maioria dos incidentes encontrados foi com } \\
\text { equipamentos e os eventos adversos observados foram } \\
\text { relativos às perdas dispositivos e alterações } \\
\text { fisiológicas. }\end{array}$ \\
\hline $\begin{array}{l}\text { Dodek et AL., } \\
2019 .\end{array}$ & $\begin{array}{l}\text { Examinar a associação entre sofrimento moral } \\
\text { em funcionários de UTI e erros de medicação e } \\
\text { eventos adversos e outros eventos adversos. }\end{array}$ & $\begin{array}{l}\text { No estudo de farmácia, quase não houve correlações } \\
\text { significativas entre sofrimento moral e medidas de } \\
\text { segurança de medicamentos. No entanto, maior } \\
\text { sofrimento moral em enfermeiros foi associado a mais } \\
\text { interceptações de quase-acidentes por erro de } \\
\text { administração, e maior sofrimento moral em médicos } \\
\text { foi associado a medições mais incorretas para } \\
\text { monitoramento de medicação por ação recomendada } \\
\text { para monitoramento. }\end{array}$ \\
\hline $\begin{array}{l}\text { Veiga VC, Postalli NF, Alvarisa } \\
\text { TK, Travassos PP, Vale RT, } \\
\text { Oliveira CZ, et al., } 2019\end{array}$ & $\begin{array}{l}\text { Descrever a incidência de eventos durante o } \\
\text { transporte intra-hospitalar de pacientes críticos } \\
\text { e analisar os fatores de risco associados. }\end{array}$ & $\begin{array}{l}\text { Transportes intra-hospitalares estão relacionados à alta } \\
\text { incidência de eventos adversos; o tempo de transporte e } \\
\text { a utilização de sedativos e drogas vasoativas estiveram } \\
\text { relacionados a esses eventos. }\end{array}$ \\
\hline $\begin{array}{l}\text { Maldonado, Ximena Alejandra; } \\
\text { Navarro Nascimento, Eliane } \\
\text { Regina Pereira do; Lzarri, } \\
\text { Daniele Delacanal. } \\
2020\end{array}$ & $\begin{array}{l}\text { Identificar como os enfermeiros relatam } \\
\text { eventos adversos; conhecer os elementos que } \\
\text { influenciam a notificação de eventos adversos e } \\
\text { quais estratégias eles sugerem para a } \\
\text { notificação. }\end{array}$ & $\begin{array}{l}\text { Os enfermeiros que notificaram os eventos adversos } \\
\text { (incluindo erros na administração de medicamentos, } \\
\text { flebite química, úlceras por pressão e auto-remoção de } \\
\text { cateteres invasivos). A não notificação de eventos } \\
\text { adversos se deve ao desconhecimento da cultura de } \\
\text { segurança e medo por punições. Como estratégias } \\
\text { sugerem educação continuada sobre a cultura de } \\
\text { segurança, que o erro levará a um plano de melhoria } \\
\text { que evita cometer o mesmo. }\end{array}$ \\
\hline $\begin{array}{l}\text { Roque KE et al., } \\
2016\end{array}$ & $\begin{array}{l}\text { Avaliar a ocorrência de eventos adversos e o } \\
\text { impacto deles sobre o tempo de permanência e } \\
\text { a mortalidade na UTI de um hospital de ensino }\end{array}$ & $\begin{array}{l}\text { Confirmados } 324 \text { eventos adversos em } 115 \text { pacientes } \\
\text { internados ao longo de um ano. A maior proporção foi } \\
\text { por úlcera por pressão, Eventos relacionados ao } \\
\text { cateteres vasculares, manejo ventilatório,cateteres } \\
\text { urinários cateter gástrico, hipoglicemia, } \\
\text { medicação/fluidos intravenosos. }\end{array}$ \\
\hline $\begin{array}{l}\text { Toffoletto MC, et AL. } \\
2016\end{array}$ & $\begin{array}{l}\text { Identificar os fatores relacionados à ocorrência } \\
\text { de eventos adversos em pacientes idosos } \\
\text { críticos internados em UTI segundo } \\
\text { características demográficas e clínicas. }\end{array}$ & $\begin{array}{l}315 \text { idosos, } 94 \text { sofreram eventos. }(60,6 \%) \text {, permanência } \\
\text { de } 10,6 \text { dias e sobreviventes }(61,7 \%) \text {. Dos } 183 \text { eventos, } \\
\text { houve predomínio do tipo processo clínico e } \\
\text { procedimento }(37,1 \%) \text {-problemas relacionados a } \\
\text { intubação, não realização de exames(glicemia), } \\
\text { cuidados com cateteres e drenos. }\end{array}$ \\
\hline $\begin{array}{c}\text { Costa TD, Salvador PTCO, } \\
\text { Rodrigues CCFM, Alves KYA, } \\
\text { Tourinho FSV, Santos VEP. } \\
2016\end{array}$ & $\begin{array}{l}\text { Compreender a percepção dos profissionais de } \\
\text { enfermagem de unidades de terapia intensiva } \\
\text { gerais de hospitais públicos acerca da segurança } \\
\text { do paciente. }\end{array}$ & $\begin{array}{l}\text { Quatro classes foram analisadas: necessidades de } \\
\text { mudança; ações diante do evento adverso; concepções } \\
\text { sobre cuidado seguro; e ações que integram o cuidado } \\
\text { seguro. Revelou-se que a segurança do paciente é uma } \\
\text { consequência da cultura adotada pelos profissionais. }\end{array}$ \\
\hline
\end{tabular}

Fonte: Autores, Recife (2021). 
A unidade de terapia intensiva (UTI) é o local que abarca pacientes graves, hemodinamicamente instáveis que necessitam de diversos procedimentos e intervenções para manejar e recuperar a saúde desses e com isso amplia o risco e a chance de ocorrer eventos adversos (EA) (Merino, 2013). Conforme a Organização Mundial da Saúde (OMS) são incidentes que atingem ao paciente e causa um dano que poderia ter sido prevenido (WHO, 2009).

Os Estudos evidenciaram que os EA que apresentam maior prevalência estão em sua maioria atrelados aos cuidados de saúde atribuído a toda equipe que assiste ao paciente, porém, os cuidados da enfermagem merecem destaque, uma vez que essa categoria profissional presta uma assistência direta nas 24 horas, tendo maior tempo realizando procedimentos, possibilitando assim a ocorrência de erros (Dutra et al.,2017; Padilha, 2006; Toffolleto et al, 2016).

As pesquisas que compuseram esta revisão ressaltaram que os EA mais prevalentes em UTI são relacionados a procedimentos/processos clínicos. Segundo estudos de Toffolleto et al. (2016) e de Roque et al. (2016) esse número corresponde a $37,1 \%$ e 59,3\% respectivamente com destaque as lesões por pressão, danos relacionados a manejos de dispositivos (tubo endotraqueal, sondas nasoenteral e vesicais de demora, não coleta de exames (ocasionando hipoglicemia), seguido das infecções relacionadas a assistência à saúde sendo (IRAS) sendo as mais recorrentes a infecção de corrente sanguínea por uso de cateter vascular e a pneumonia associada a ventilação mecânica.

Não raro, eventos relativos a erros de medicações são frequentes cujos valores correspondem de $2,5 \%$ a $13,8 \%$, aumentando a chance de sua ocorrência, sendo proporcional ao quantitativo de medicações (cinco e mais) usadas pelo paciente (Dutra et al.,2017; Dodek et al., 2019; Roque et al.,2016; Toffolleto et al.,2016).

Silva et al.(2018), em sua pesquisa abordando ocorrência de incidentes e eventos adversos relacionados ao transporte intra-hospitalar de pacientes internados em uma Unidade de Terapia Intensiva de um hospital da região Sudeste do Brasil, relatou que a ocorrência de eventos em pacientes críticos pode ocorrer também durante o transporte intra-hospitalar na vigência da necessidade de realizar algum exame ou procedimento não disponível no setor UTI.

Corroborando com o estudo anterior, segundo Kleffmann et al.(2016); Veiga et al.(2019), os eventos mais recorrentes durante esse transporte são: perda de dispositivos (extubação acidental, perda do cateter venoso, cateter de oxigênio e cateter vesical), instabilidade hemodinâmica, aumento ou queda da pressão arterial média, dessaturação e falhas com equipamentos (término de bateria de bombas de infusão, término do gás oxigênio.

Existem múltiplos fatores que propiciam a ocorrência de EA no transporte intra-hospitalar cujos principais são: uso de sedativos, drogas vasoativas, maior tempo de transporte (superior a 30 minutos), falta de comunicação entre setores e equipes (Silva et al., 2018; Alamanou et al.,2017; Veiga et al.,2019). Acrescento que muitos incidentes com danos ocorrem durante o transporte interssetores por comunicação não efetiva, não divisão de funções aos membros de compõem a equipe e ausência de um check list que oriente quais os passos a serem seguidos e os materiais necessários para o procedimento a fim de que não haja esquecimento e por fim o erro.

A fim de se prevenir a ocorrência desses eventos é necessária uma equipe capacitada e treinada, prover equipamentos e materiais com antecedência ao transporte, prever possíveis complicações e manter boa comunicação entre os membros da equipe de transporte, que relativo ao paciente crítico deve ser composta por Enfermeiro, técnico e médico habilitados a fim de se garantir um procedimento seguro, livre de danos aos pacientes (Silva et al., 2018; Veiga et al., 2019; Rodríguez et al., 2020).

As pesquisas destacam também um conjunto complexo de fatores para o surgimento de um evento adverso dentro de uma UTI, que corroboram para a gravidade do caso, maior tempo de internação hospitalar e desfechos negativos ao cliente. Os fatores mais prevalentes são: idade, sexo, comorbidades, gravidade, maior carga de trabalho da enfermagem, cargas horárias extensivas, desproporcional dimensionamento de pessoal, despreparo da equipe assistencial (Roque et al., 2016; Costa El at., 2016; Toffolleto et al., 2016; Dutra et al., 2017; Rodríguez et al., 2020; Kelly, 2014; Gonçalves et al.,2006). 
Relativo a esses fatores existem controvérsias entre os estudos. As pesquisas de Roque et al.(2016) e Toffolleto et al.(2016) corroboram que existe uma associação entre a idade e a maior chance de ocorrer um evento adverso, em contrapartida Veiga et al.(2019) afirma que este fator não se associa ao acontecimento do incidente.

Entretanto os estudos desta revisão evidenciam que quanto maior tempo de internação pela maior exposição e conforme maior gravidade do estado clínico do paciente há maior chance de ocorrência do EA. Não raro tendo-se um dimensionamento de pessoal inadequado e os fatores anteriormente citados estes contribuem também para um maior tempo de trabalho da enfermagem dedicada a este cliente (Roque et al.,2016; Toffolleto et al.,2016; Rodríguez et al.,2020; Kelly, 2014; Gonçalves et al.,2006).

A fim de se garantir a segurança do paciente com uma assistência de saúde livre ou com o mínimo de danos, através da cultura de segurança do paciente são eleitas estratégias. Os estudos afirmam que a principal delas corresponde à notificação do evento, pois, por meio do conhecimento do incidente é possível refletir e traçar medidas para prevenir ou reduzir novos eventos da mesma natureza (Teodoro et al.,2020; Costa et al.,2016; Roque et al.,2016; Kelly, 2014; Gonçalves et al.,2006).

Como estratégias de prevenção de eventos adversos, além da notificação Costa et al.(2016) afirma em seu estudo que propõe compreender a percepção dos profissionais de enfermagem de UTIs gerais acerca de segurança do paciente e Teodoro et al.(2020) que em sua pesquisa traz a importância de se notificar incidentes como uma forma de propor cultura de segurança do paciente para melhoria assistencial, trazem a sensibilização da equipe acerca de mudar a forma de pensar em prol da cultura de segurança do paciente promovendo cuidados de saúde baseados nas melhores evidências científicas.

Costa et al.(2016) e Teodoro et al.(2020) ressaltam também a importância de buscar conhecer a equipe de saúde e suas dificuldades assistenciais diárias a fim de promover treinamentos que intervenham sobre os processos de trabalho; investir em educação permanente hospitalar que propõe atualização, especialização e melhoria na qualidade do atendimento; confecção e validação de check lists e protocolos assistenciais e também o levantamento de indicadores relativos aos eventos para uma mudança na conduta assistencial para prevenção de novos eventos adversos.

Porém, existem obstáculos a respeito da notificação de EA. Maldonado et al. (2020) e Mansouri et al.(2019) relatam que existe, por parte da equipe o medo de punição, reclamações, descontos nos salários, advertências. Deve-se salientar que o real intuito da notificação está em promover melhorias da assistência ao paciente sem apontar culpados.

Foram identificadas como limitações dessa revisão integrativa, que embora os eventos adversos sejam considerados um problema de saúde pública bem presentes nas unidades de terapia intensiva nem todos os estudos trouxeram possível estratégias preventivas dos Eventos adversos. Verificou-se que ainda existem poucos estudos que retratem acerca das principais medidas e estratégias práticas a serem usadas para prevenção, sugerindo-se assim que sejam publicados novos estudos acerca da temática em questão trazendo novas evidências que contribuam para as práticas assistenciais de saúde.

\section{Considerações Finais}

Esta revisão traz como principais achados que a ocorrência de Eventos adversos está associada a múltiplos fatores atrelados ao paciente (Idade, maior tempo de internação), à gravidade (quadro clínico do paciente, grande quantitativo de dispositivos e drogas) e aos processos de trabalho (inadequado dimensionamento, carga horária extensiva e despreparo assistencial da equipe). Frente a isso se evidencia que a fim de garantir a segurança do paciente é consenso que a assistência à saúde deve ser livre de danos através da implementação de diversas medidas.

Foi evidenciado nos estudos também que as medidas e estratégias implementadas após o processo de notificação como a educação permanente, estabelecimento de protocolos assistenciais e levantamento de indicadores tiveram por objetivo aprimorar os processos de trabalho da assistência à saúde para que novos eventos não acontecessem novamente, sem carácter punitivo a fim de promover a melhora clínica do cliente e um atendimento humanizado. 


\section{Referências}

2017, 7(2):177-87. http://www.hsj.gr/medicine/interruption-oftherapy-during-intrahospital-transport-of-non 8208icu-patients.pdf),

Brasil. Agência Nacional de Vigilância Sanitária. Medidas de Prevenção de Infecção Relacionada à Assistência à Saúde/Agência Nacional de Vigilância Sanitária. Anvisa, 2017. http://portal.anvisa.gov.br/ >

Brasil. Anvisa. Gestão de Riscos e Investigação de Eventos Adversos Relacionados à Assistência à Saúde. < http://portal.anvisa.gov.br/ >

Brasil. Ministério da Saúde. Agência Nacional de Vigilância Sanitária. RDC ANVISA/MS no 36, de 25 de julho de 2015 . Institui ações para a segurança do paciente em serviços de saúde e dá outras providências [Internet]. Brasília: Ministério da Saúde, 2015 <http://portal.anvisa.gov.br/documents/10181/2871504/RDC_36_2013_COMP.pdf/36d809a4e5ed-4835-a375-3b3e93d74d5e 4>

Brasil. Ministério da Saúde. Gabinete do Ministro. Portaria GM/MS no 529, de 1 de abril de 2013. Institui o Programa Nacional de Segurança do Paciente (PNSP) [Internet]. Brasília: Ministério da Saúde, 2013: http://bvsms.saude.gov.br/bvs/saudelegis/g m/2013/prt0529_01_04_2013.html

CASP. Critical Appraisal Skills Programme. http://www.casp-uk.net/casp-tools-checklists.2013

Costa, T. D., Salvador, P. T. C. O., Rodrigues, C. C. F. M., Alves, K. Y. A., Tourinho, F. S. V. \& Santos, V. E. P. Percepção de profissionais de enfermagem acerca de segurança do paciente em unidades de terapia intensiva. Rev Gaúcha Enferm.,37(3):e61145. http://dx.doi.org/10.1590/1983- 1447.2016.03.61145.

Crossetti, M. G. O. Revisão integrativa de pesquisa na enfermagem o rigor científico que lhe é exigido. Ver. gaúcha enferm. 2012, 33(2):8-9.

Dodek, P., Norena, M., Ayas, N., Dhingra, V., Brown, G. \& Wong, H. Moral distress in intensive care unit personnel is not consistently associated with adverse medication events and other adverse events. J Crit Care. 53:258-263. 10.1016/j.jcrc.2019.06.023.

Dutra, D. D., Duarte, M. C. S., Albuquerque, K. F. et al. Eventos adversos em Unidades de Terapia Intensiva: estudo bibliométrico. Rev Fund Care Online. 9(3):669-675. http://dx.doi.org/10.9789/2175- 5361.2017.v9i3.669-675

Françolin, et al. Gerenciamento da segurança do paciente sob a ótica dos enfermeiros. Rev Esc Enferm USP. 49(2):277-83. http://dx.doi.org/10.1590/S0080623420150000200013. http://www.scielo.br/pdf/reeusp/v49n2/pt_0080-6234-reeusp-49-02-0277.pdf

Gonçalves, L. A., Garcia, P. C., Toffoleto, M. C, Telles S. C. R., \& Padilha, K. G. [The need for nursing care in Intensive Care Units: daily patient assessment according to the Nursing Activities Score (NAS)]. Rev Bras Enferm. http://www.scielo.br/ pdf/reben/v59n1/a11v59n1.pdf

Jhak, et al. The global burden of unsafe medical care: analytic modelling of observational studies. BMJ Qual Saf. 22(10):809-15. https://qualitysafety.bmj.com/content/22/10/809>

Kelly, D. M., Kutney-Lee, A., McHugh, M. D., Sloane, D. M. \& Aiken, L. H. Impact of critical care nursing on 30-day mortality of mechanically ventilated older adults. Crit Care Med. 42(5):1089-95. https://www.ncbi.nlm.nih.gov/pmc/articles/PMC3993087/ pdf/nihms570089.pdf,

Kleffman, J., Pahl, R., Deinsberger, W., Ferbert, A. \& Roth, C. Intracranial pressure changes during intrahospital transports of neurocritically ill patients. Neurocrit Care. 25(3):440-5. 10.1007/s12028-016-0274-6

Knaus, W. A., Zimmerman, J. E., Facp, W., Douglas P., Draper, E. A., Lawrence, D. E. Apache - fisiologia aguda e avaliação crônica da saúde: um sistema de classificação com base fisiológica, Critical Care Medicine: 9, 591-597

Lima, C. S. P. \& Barbosa, S. F. F. Ocorrência de eventos adversos como indicadores de qualidade assistencial em unidade de terapia intensiva. Rev Enferm UERJ. 23(2):221-8. http://dx.doi.org/10.12957/reuerj.2015.6076. http://www.facenf.uerj.br/v23n2/v23n2a13.pdf.

Maia, C. S., et al. Notificação de eventos adversos relacionados com a assistência à saúde que levaram a óbitos no Brasil 2014-2016. Epidemiol. Serv. Saude, 27(2):e2017320. http://www.scielo.br/scielo.php?script=sci_arttext\&pid=S2237-96222018000200308\&lang=pt

Maldonado, N. X., Nascimento, E. R. P. \& Lazzari, D. D. Nursing professionals and adverse event reporting. Texto Contexto Enferm. 29 (Spe): e20190282. https://doi.org/10.1590/1980-265X-TCE-2019-0282

Mansouri, S. F., Mohammadi, T. K., Adib, M., Lili, E. K. \& Soodmand, M. Barriers to nurses reporting errors and adverse events. Br J Nurs. $28(11): 690-695$. 10.12968/bjon.2019.28.11.690.

Merino, P. et al. Study Investigators. Adverse events in Spanish intensive care units: the SYREC study. Int J Qual Health Care. 24:105-13. 4. https://www.ncbi.nlm.nih.gov/pubmed/22190588

Nazareno, R. M. P. F. et al. Ocorrências iatrogênicas nas Unidades de Terapia Intensiva: enfoque nas ações da equipe de enfermagem. Rev eletrônica enferm. 1 (1):1-16. 24. http://www.scielo.br/pdf/rlae/v9n5/7804.pdf

Novaretti, M. C. Z. et al. Sobrecarga de trabalho da Enfermagem e incidentes e eventos adversos em pacientes internados em UTI. Rev Bras Enferm. 67(5):692-9. http://www.scielo.br/scielo.php?pid=S0034-71672014000500692\&script=sci_abstract\&tlng=pt

Oxford. Centre for evidence-based medicine:levels of evidence. http://www.cebm.net/oxford-centre-evidence-levels-evidence-march-2009

Padilha, K. G. Ocorrências iatrogênicas em unidade de terapia intensiva (UTI): análise dos fatores relacionados. Rev Paul Enferm. 25 (1).

Pagnamenta, A. \& et al. Adverse event reporting in adult intensive care units and the impact of a multifaceted intervention on drug-related adverse events. Ann Intensive Care. 2012, 2:47. https://www.ncbi.nlm.nih.gov/pubmed/23174137 
Pompeo, D. A., Rosssi, L. A, \& Galvão, C. M.(2009). Revisão integrativa: etapa inicial do processo de validação de diagnósticos de enfermagem. Acta Paulista de enfermagem, 22(4):434-8. http://doi.org/10.1590/sd103-2100200900040014.

Rodríguez, M. F., López-Izquierdo, R., Mohedano-Moriano, A., Polonio-López, B., Maestre Miquel, C., Viñuela, A., Fernández, C. D., Correas, J. G., Marques, G. \& Martín-Conty, J. L. Identification of Serious Adverse Events in Patients with Traumatic Brain Injuries, from Prehospital Care to Intensive-Care Unit, Using Early Warning Scores. Int J Environ Res Public Health. 17(5):1504. 10.3390/ijerph17051504.

Roque, K. E. \& et al. Eventos adversos na unidade de terapia intensiva: impacto na mortalidade e no tempo de internação em um estudo prospectivo. Cad. Saúde Pública, 32(10):e00081815, http://dx.doi.org/10.1590/0102-311X00081815. http://www.scielo.br/scielo.php?script=sci _arttext\&pid=S0102$311 \mathrm{X} 2016001005001 \& \operatorname{lng}=$ en \&nrm=iso.

Runciman, W. \& et al.Towards an international classification for patient safety: key concepts and terms. Int J Qual Health Care. 21:18-26. 11. https://academic.oup.com/intqhc/article/21/1/18/1888152>

Silva, R., Amante, L. N., Salum, N. C. \& et al. Incidentes e Eventos Adversos no Transporte Intra-Hospitalar em Terapia Intensiva. 2018,8:e2805. http://dx.doi.org/10.19175/recom.v8i0.2805

Souza, V. R. \& et al. O estresse de enfermeiros atuantes no cuidado do adulto na unidade de terapia intensiva. $J$ res fundam care online. $25-8$. zhttp://www.seer.unirio.br/index.php/cuidadofundamental/article/view/1687/pdf_501 >

Teodoro, R. F. B., Silva, A. S., Carreiro, M. A., Bilio, R. L. \& Paula, D. G. Análise da notificação de eventos adversos através da pesquisa de cultura de segurança do paciente. Rev Fun Care Online. 12:463-470. http:// dx.doi.org/10.9789/2175-5361.rpcfo.v12.8521

Toffolleto, M. C., Barbosa, R. L., Andolhe, R., Oliveira, E. M., Ducci, A. J. \& Padilha, K. G. Factors associated with the occurrence of adverse events in critical elderly patients. Rev Bras Enferm 2016,69(6):977-83. http://dx.doi.org/10.1590/0034-7167-2016-0199

Veiga, V. C., Postalli, N. F., Alvarissa, T. K. et al. Eventos adversos durante transporte intra-hospitalar de pacientes crítico. Rev Bras Ter Intensiva. 2019,31(1):15-20.

Whittemore, R. \& Knaff, K. Revisão integrativa review: update methodology. Leading global nursing research, Ingaterra, 52(5):546-553, 2005

Who. World Health Organization. Summary of the evidence on patient safety: implications for research. Edição: AshishJha, https://pesquisa.bvsalud.org/portal/resource/pt/who-43874

World Health Organization (WHO). The conceptual framework for the international classification for patient safety v1.1: final technical report and technical annexes. http://www.who.int/patientsafety/taxonomy/icps_full_report.pdf 